\title{
Carnets
}

Revue électronique d'études françaises de l'APEF

Deuxième série - 16 | 2019

Le Récit inachevé: études sur Mai 68

\section{Mai-68... et après ? Une nouvelle donne politique. La France de 1962 à 1984}

\section{Gilles Richard}

\section{Q OpenEdition}

1 Journals

\section{Édition électronique}

URL : http://journals.openedition.org/carnets/9599

DOI : 10.4000/carnets.9599

ISSN : 1646-7698

Éditeur

APEF

Référence électronique

Gilles Richard, «Mai-68... et après ? Une nouvelle donne politique. La France de 1962 à 1984 », Carnets [En ligne], Deuxième série - 16 | 2019, mis en ligne le 31 mai 2019, consulté le 10 novembre 2019. URL : http://journals.openedition.org/carnets/9599; DOI : 10.4000/carnets.9599

Ce document a été généré automatiquement le 10 novembre 2019.

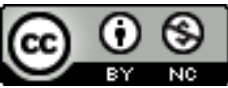

Carnets est mis à disposition selon les termes de la licence Creative Commons - Atribution - Pas d'utilisation commerciale 4.0 International. 


\title{
Mai-68... et après ? Une nouvelle donne politique. La France de 1962 à 1984
}

\author{
Gilles Richard
}

1 Pour le $50^{e}$ anniversaire de 1968, l'Association portugaise d'études françaises (APEF) a choisi de consacrer les deux journées de son Forum annuel, les 11 et 12 juin 2018, aux « événements de 68 » en France. Immense sujet sur lequel plus d'un millier d'ouvrages de tous types sont parus en un demi-siècle : essais, témoignages, études universitaires, publications de documents, numéros spéciaux de revues, de journaux quotidiens ou périodiques $^{1}$; sans compter les expositions et leurs catalogues ${ }^{2}$, les conférences, les rétrospectives radiodiffusées ou télévisées, les films documentaires ${ }^{3}$ ou de fiction ${ }^{4}$.

2 Face à une telle masse de matériaux, l'historien que je suis ne peut qu'être frappé par l'écart considérable qui existe entre la réalité des faits tels qu'ils se sont déroulés d'une part, la mémoire collective dominante qui en est conservée d'autre part. Dans la France d'aujourd'hui, une vision rétrospective s'impose en effet avec la force de l'évidence : le Mai-68 français n'aurait été qu'un épisode d'un 68 global pensé comme un mouvement planétaire de contestation socio-culturelle impulsé par la jeunesse scolarisée des grandes villes. Certes, les contestations étudiantes existèrent bel et bien, puissantes dans nombre de pays. Les "années 1968 " furent une période où «la jeunesse " se montra prompte à rejeter "l'impérialisme» dans le contexte des décolonisations largement entamées en Asie et en Afrique - l'empire portugais connaissant une situation quelque peu décalée dans le temps alors que la France avait perdu l'essentiel de ses colonies entre 1960 et 1962 . Cela se traduisit de part et d'autre de l'Atlantique mais aussi en Asie et même dans quelques pays d'Afrique comme le Sénégal, par d'innombrables manifestations contre la guerre du Viêt-Nam et l'apartheid en Afrique $\mathrm{du}$ Sud ainsi que par une grande attention portée au problème du "sousdéveloppement " et une sorte de culte voué à l'image héroïque d'Ernesto "Che » Guevara. Mais de là à prétendre que tous les pays ou presque vécurent une situation comparable, il y a un pas qu'il me paraît difficile de franchir, les transferts 
d'expériences, fort complexes par nature et au demeurant encore mal connus, n'effaçant pas les spécificités des différentes sociétés concernées. Ainsi, les maoïstes français, peu nombreux mais très actifs et médiatisés, déjà engagés dans "l'établissement" en usines ${ }^{5}$ pour y développer la conscience révolutionnaire des masses ouvrières à leurs yeux anesthésiées par les «bureaucrates» et les "réformistes", n'avaient aucun rapport réel avec les Gardes rouges, lycéens en majorité, armés par Mao Zedong et Lin Biao pour éliminer par la force les cadres réputés "droitiers" au sein du Parti communiste chinois. De même, les étudiants tchécoslovaques qui refusaient le stalinisme et aspiraient à la démocratisation de leur pays grâce à l'instauration d'élections libres et d'un système partisan pluraliste, n'étaient manifestement pas sur la même longueur d'onde que bon nombre de leurs homologues français critiquant à l'envi la «démocratie bourgeoise » et qualifiant les élections de " piège à cons ».

3 Bien des ressemblances soulignées, dans une vision "globalisante » à la mode de nos jours, entre les événements survenus dans divers pays en 1968 ne sont en fait que partielles, voire superficielles et, quoi qu'il en soit, les connexions d'un pays à l'autre bien réelles parfois: les liens entre SDS ouest-allemand (Sozialistischer Deutscher Studentenbund) et JCR française (Jeunesse communiste révolutionnaire) par exemple ne peuvent à l'évidence tout expliquer. Et surtout pas les événements survenus en France dont la spécificité fut particulièrement nette.

4 La France est un pays où l'on aime commémorer le passé ; « 68 » n'échappe pas à la règle. Notons toutefois que rien ou presque n'eut lieu en 1978, année d'échec des gauches désunies aux élections législatives. Dix ans plus tard en revanche, année qui vit François Mitterrand réélu président de la République, parut aux éditions du Seuil le livre d'Hervé Hamon et Patrick Rotman, Génération, qui connut un très grand succès. Il braquait les projecteurs de la mémoire sur quelques dizaines d'intellectuels parisiens qui pour la plupart avaient, vingt ans après les événements, trouvé leur place au sein des classes dirigeantes, à commencer par Serge July et Daniel Cohn-Bendit. Plus largement, l'ouvrage donnait de 68 l'image d'un mouvement avant tout étudiant dont l'épicentre aurait été le Quartier latin à Paris. En 1998, l'appréhension des faits commença toutefois à évoluer grâce aux premiers colloques d'historiens organisés en lien avec les services d'archives et la BDIC (Bibliothèque de documentation internationale contemporaine) $)^{6}$. Mais ce fut surtout en 2008 que la vague mémorielle prit des dimensions impressionnantes (plus de 300 ouvrages en un $a^{7}$ ), portée par les effets de la campagne présidentielle de 2007 durant laquelle Nicolas Sarkozy avait fait de la liquidation de «l'héritage de 68 » un des principaux points de son programme. L'année 2018 s'inscrit dans le prolongement des travaux publiés depuis dix ans, mettant désormais davantage l'accent sur la publication de témoignages d'acteurs ordinaires $^{8}$ et analysant les événements de façon fine à l'échelon des régions et des villes de province?.

5 Avec 50 ans de recul, on peut donc maintenant considérer comme définitivement assuré, même si la mémoire collective dominante ne l'a pas encore pleinement intégré, le fait que 68 ne s'est pas réduit en France à des combats de rue entre étudiants et policiers au Quartier latin - à propos desquels les commentateurs continuent de s'étonner qu'ils n'aient pas fait de mort et de louer la sagesse du préfet de police Maurice Grimaud. Or, 68 fut un événement à la fois national et d'une grande violence : sept morts (tous en dehors du Quartier latin) en deux mois et plus de 8000 blessés dont 
des dizaines très gravement (œil perdu, membre amputé, traumatisme crânien avec séquelles, etc.). Et l'aspect le plus important ne fut pas tant le mouvement de contestation des étudiants que le déclenchement d'une grève générale d'une ampleur jamais vue en Europe, sept millions de grévistes occupant les entreprises quatre semaines durant. Aucun autre pays, l'Italie mise à part avec son « Mai rampant » décalé dans le temps, ne connut une telle situation.

6 Sur la base de ce constat aujourd'hui solidement documenté par les historiens et l'ensemble des chercheurs en sciences sociales, quatre points méritent à mon sens d'être précisés.

\section{Mai-68 a commencé avant mai 1968}

7 La contestation de l'ordre établi n'a pas débuté le 3 mai avec la manifestation étudiante boulevard Saint-Michel suite à l'évacuation de la Sorbonne par la police, ni même le 22 mars avec l'occupation du bâtiment de la direction de l'université de Nanterre, mais bien plutôt en 1965-1966 avec les protestations contre le règlement des cités universitaires non mixtes et la critique publique d'un système pédagogique devenu inadapté à la massification des universités. Parallèlement, le monde salarié entra dans un cycle décennal d'insubordination dans le cadre du pacte d'unité d'action signé entre la CGT et la CFDT en 1966. Dès 1967, le nombre des journées de grèves dépassa le seuil des quatre millions, conduites notamment contre les ordonnances gouvernementales qui s'attaquaient pour la première fois de manière frontale à la Sécurité sociale ${ }^{10}$. Impossible dans ces conditions d'analyser Mai-68 comme un processus historique déclenché par les étudiants et auquel les ouvriers se seraient ralliés au milieu du mois de mai. Des luttes sociales tous azimuts étaient déjà bien engagées, les salariés ayant leurs propres revendications et leur propre calendrier de manifestations. Ainsi, une série d'actions régionales unitaires "tournantes" était programmée pour avril-mai 1968 - le 8 mai, un jour calme dans le Quartier latin, l'Ouest de la France connut des défilés au cri de « l'Ouest veut vivre ", d'une ampleur jamais vue encore, y compris dans des petites villes comme Pontivy, Redon, Lamballe ou Landerneau ${ }^{11}$.

Exemple emblématique des luttes ouvrières, la grève déclenchée dès la fin du mois d'avril à l'usine Sud-Aviation de Bouguenais, près de Nantes, pour des questions de salaires et de conditions de travail. Après trois semaines de conflit et face à l'intransigeance de la direction parisienne du groupe (Maurice Papon, ancien préfet de police de Paris, avait pris la tête de l'entreprise publique Sud-Aviation en 1967), les 2000 salariés de l'usine, voyant le succès de la grève nationale du 13 mai en soutien aux étudiants victimes des violences policières et ayant participé, au moins pour certains d'entre eux, à la nuit d'émeute qui avait suivi jusque dans les jardins de la préfecture de Loire-Atlantique à Nantes, décidèrent le 14 au matin d'occuper les locaux et de séquestrer - « retenir » dans le langage syndical - les cadres dirigeants de l'usine. Cette occupation inspira dès le lendemain plusieurs usines en lutte dans la région du Havre, rejointes le 16 par les 35000 ouvriers de l'usine Renault de Boulogne-Billancourt, la plus grande usine de France alors. Une semaine plus tard, près de sept millions de salariés étaient en grève. Difficile, on le voit, dans ces conditions de prétendre que les manifestations étudiantes du Quartier latin furent à l'origine de la grève générale. On assista plutôt à la mi-mai à un processus de coagulation de luttes diverses et déjà 
engagées, dans une crise sociale générale, débouchant en quelques jours sur une crise politique qui poussa Charles de Gaulle à interrompre son voyage officiel en Roumanie.

\section{Y eut-il pour autant « vacance du pouvoir »?}

Ce fut ce que prétendit dans sa conférence de presse du 28 mai François Mitterrand, candidat d'union des gauches à l'élection présidentielle de 1965 et dirigeant de la FGDS (Fédération de la gauche démocrate et socialiste), une organisation qui regroupait les forces de «la gauche non communiste » comme on disait alors. Face à la paralysie du pays, il pronostiqua la démission prochaine du chef de l'État, annonça qu'il serait candidat à sa succession et désigna par avance Pierre Mendès France comme son futur Premier ministre! Mais tout démontre aujourd'hui que loin d'être vacant, le pouvoir gaulliste, avant même que Charles de Gaulle partît à Baden-Baden chercher le soutien des chefs de l'armée et revînt triomphalement le 30 mai, était toujours en place. Georges Pompidou fut sans conteste la figure centrale de la résistance à la contestation généralisée. Il défendit sa politique à l'Assemblée nationale, rencontra plusieurs dirigeants centristes, organisa la négociation avec les syndicats, pesa de tout son poids pour que le président de la République reportât sine die le référendum qu'il avait annoncé le 24 mai, manœuvra pour accélérer la reprise du travail dans la Fonction publique début juin et supervisa personnellement la préparation des élections législatives des 23 et 30 juin - elles furent un véritable triomphe pour le parti gaulliste dont le Premier ministre avait pris le contrôle depuis 1967.

Tout cela se fit néanmoins - sans qu'on l'ait bien saisi sur le moment - sur la base d'une rupture définitive entre Charles de Gaulle et Georges Pompidou. Chacun analysait alors la situation à sa façon. Pour le premier, il fallait rétablir l'ordre par tous les moyens, y compris militaires, mais engager ensuite, sans tarder, une profonde réforme des entreprises en y instaurant «la participation » de tous les salariés à leur gestion. Pour le second au contraire, il fallait arrêter la grève générale en jouant sur les divisions des adversaires du pouvoir et en faisant un certain nombre de concessions (ce fut l'objet des négociations au ministère du Travail, rue de Grenelle, du 25 au 27 mai) mais il n'était pas question de la moindre « participation » qui aurait porté gravement atteinte au pouvoir patronal dans les entreprises.

11 C'est seulement sur la base de cette rupture politique entre les deux hommes que l'on peut comprendre les événements du printemps 1969 : échec du référendum voulu par Charles de Gaulle (dès le début de la campagne référendaire, son ancien Premier ministre se posa ouvertement en successeur tandis que Valéry Giscard d'Estaing appelait publiquement à voter "non») puis élection de Georges Pompidou à l'Élysée, avec le soutien du même Valéry Giscard d'Estaing qui retrouva en échange le ministère des Finances, perdu en 1966 sur pression des gaullistes de gauche.

On le voit, l'étude de « 68 » ne peut se réduire à la seule période de mai et juin 1968. La contestation du pouvoir gaulliste et de l'organisation de la société, commencée avant 1968 , se poursuivit bien après. 


\section{De 1968 à 1974, espoir ou crainte d'un nouveau Mai-68}

13 S'en tenir au constat, souvent répété, d'une contradiction entre la plus grande grève générale de l'histoire nationale (et européenne) déclenchée le 13 mai 1968 débouchant le 30 juin sur la plus grande victoire électorale des droites depuis les débuts de la $\mathrm{III}^{\mathrm{e}}$ République (qu'un groupe parlementaire - le groupe gaulliste en l'occurrence - eût à lui seul la majorité des sièges au Palais-Bourbon ne s'était jamais vu) ne peut suffire à comprendre l'enjeu de ces huit semaines évoquées précédemment. C'est qu'un si puissant mouvement social produit nécessairement des effets à moyen et long termes que l'on ne pouvait sur le moment envisager. Plusieurs semaines de perturbation du quotidien permirent ce que Michelle Zancarini-Fournel et Xavier Vigna ont appelé des « rencontres improbables». Rencontres tout d'abord entre jeunes ouvriers et jeunes étudiants, souvent côte à côte dans les manifestations à Paris et en province, ou dans les occupations d'établissements publics, à commencer par la Sorbonne; rencontres aussi entre ouvriers en grève et paysans solidaires venant leur apporter de la nourriture gratuitement - il en sortit le mouvement des "paysans travailleurs" de Bernard Lambert, à l'origine de l'actuelle Confédération paysanne; rencontres enfin entre ouvriers, techniciens, cadres et ingénieurs dans plusieurs dizaines de grandes usines occupées où l'on échangea longuement sur les nouvelles façons de gérer les entreprises et où émergea l'aspiration à «l'autogestion » qui allait marquer bien des conflits sociaux des années suivantes.

14 À moyen terme, les conséquences de ces "rencontres improbables " mais aussi des contradictions apparues durant les événements furent considérables. Soulignons d'abord l'essor de ce que les historiens nomment la deuxième vague féministe. Dès 1970 , les femmes engagèrent un combat sans concession bien que pacifique sur les questions de la contraception et du droit à l'avortement - « un enfant si je veux, quand je veux $»^{12}$. Émergea aussi l'écologie politique, d'abord portée par de multiples associations de défense de la nature. Les mouvements régionalistes prirent leur essor: Corse, Bretagne, Alsace, Pays basque, "Occitanie ». Autant d'aspects qui reçurent leur traduction politique lors de l'élection présidentielle de 1974: première femme candidate (Arlette Laguiller), premier candidat écologiste (René Dumont), premiers candidats pour une Europe des régions (Guy Héraud et Jean-Claude Sebag). Plus largement, le système partisan amorça une évolution d'ampleur. Durant les «Trente Glorieuses ", les gaullistes à droite et les communistes à gauche avaient dominé la vie politique; à compter du milieu des années 1970, les libéraux, sous la conduite de "VGE", et les socialistes, sous celle de François Mitterrand, s'imposèrent progressivement sur leurs partenaires respectifs ${ }^{13}$ : en 1981, les deux hommes s'affrontèrent au second tour de l'élection présidentielle. Insistons enfin sur le fait qu'en profondeur, les valeurs traditionnelles de la société, en lien souvent avec celles de l'Église, se trouvèrent remises en cause : l'autorité, la nation associée à l'armée (le long combat contre l'extension du camp militaire du Larzac débuta en 1972), la famille, le travail.

15 Sur ce dernier point, le patronat organisé, c'est-à-dire le CNPF (Conseil national du patronat français), se montra particulièrement perspicace, mesurant sur-le-champ le danger qu'il y avait à laisser contester l'organisation capitaliste taylorisée des entreprises. 


\section{Le CNPF à l'avant-garde de la contre-offensive des classes dirigeantes}

16 C'est sans doute l'aspect le moins connu du «moment 68 » que la façon dont le grand patronat réagit à la grève générale. Sur le moment, il s'en remit pour l'essentiel au gouvernement de Georges Pompidou. Mais dès l'automne, une réflexion de fond s'engagea pour chercher les moyens d'endiguer la contestation de l'ordre économique. La figure centrale autour de laquelle une stratégie de contre-offensive s'élabora fut sans conteste François Ceyrac, alors vice-président du CNPF et président de la commission sociale de l'organisation.

17 Entré en 1936 au service du patronat comme "permanent " chargé de rédiger les conventions collectives pour l'UIMM (Union des industries métallurgiques et minières), il avait acquis une excellente connaissance des syndicats de salariés et noué des relations étroites avec le secrétaire général de Force ouvrière (FO), André Bergeron, tout en étant très proche de Georges Pompidou dont il avait été le condisciple au lycée Louis-le-Grand à la fin des années 1920. L'autogestion était le danger principal aux yeux des patrons. Comment empêcher les salariés, des ouvriers jusqu'aux cadres, de réclamer le contrôle de la gestion des entreprises, et comment enrayer la vague des grèves qui s'amplifia à nouveau après une brève accalmie en 1969-1970, jetant dans les luttes de plus en plus de femmes et d'immigrés?

Sans qu'il soit ici possible d'entrer dans tous les détails de la stratégie mise au point ${ }^{14}$, disons que le patronat organisé, derrière François Ceyrac devenu président du CNPF en 1972 à la place de Paul Huvelin, choisit d'entrer directement dans le combat pour défendre «l'entreprise ». Lors de l'émission "À armes égales » sur la deuxième chaîne le 27 octobre 1970, François Ceyrac accepta d'affronter en direct durant deux heures le secrétaire général de la CGT, Georges Séguy, sur le thème de la grève : jamais jusque-là un responsable patronal n'avait en France accepté une telle chose. On expérimenta dans les entreprises une série de réformes dans les modes de gestion des salariés: nouveau «management» des cadres, recours à l'intérim pour les jeunes et au temps partiel pour les femmes, etc. Mais à plus long terme, ce fut le taylorisme dans son ensemble qui fut remis en cause, remplacé par une nouvelle organisation du travail en équipes réduites et collectivement responsables tandis que les prodromes de ce que l'on n'appelait pas encore la révolution numérique - on parlait alors d'« automation » permit de réduire le nombre des ouvriers nécessaires, entraînant par contrecoup l'augmentation du chômage.

19 De ce combat quotidien du patronat organisé pour défendre l'organisation économique capitaliste, livré pied à pied, jour après jour avec le soutien des gouvernants, quasiment officiel à partir de l'arrivée de Raymond Barre à Matignon, le mouvement ouvrier qui s'était construit dans les luttes depuis les années 1880 fut la principale victime. Dès 1977-1978, les syndicats commencèrent à perdre des adhérents, sans jamais pouvoir redresser la situation. Certes, en 1981, les gauches conquirent le pouvoir: François Mitterrand entra à l'Élysée et Pierre Mauroy prit la tête d'un gouvernement d'union, comportant quatre ministres communistes. Mais cela survint dans un contexte défavorable : outre l'affaiblissement des syndicats de salariés déjà bien entamé, le PCF était lui aussi entré dans un processus de déclin irrémédiable tandis que dans les grands pays comparables à la France s'imposaient des dirigeants ouvertement acquis au 
néolibéralisme (Margaret Thatcher au Royaume-Uni en 1979, Ronald Reagan aux ÉtatsUnis en 1980, Helmut Kohl en RFA en 1982). Dès 1983, les socialistes au pouvoir renoncèrent à leur programme de transformation sociale et en 1984, le remplacement de Pierre Mauroy par Laurent Fabius scella la marginalisation des communistes, au moment même où s'amorçait la montée en puissance du Front national ${ }^{15}$.

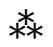

De ce 68 français évoqué ici à grands traits, quelles « leçons » retenir - si tant est que l'histoire puisse en donner? J'en soulignerai deux, contradictoires à l'image des événements décrits.

La première «leçon" est économique. On peut, dans un grand pays industriel, augmenter le salaire minimum (il fut créé en 1950, le SMIG - salaire minimum interprofessionnel garanti - et devint le SMIC - de croissance - en 1970) de $35 \%$ d'un seul coup sans provoquer un effondrement économique. Au contraire pourrait-on même dire, puisque le taux de croissance du PIB français connut ses plus hauts niveaux (6 à $7 \%$ par an en moyenne) durant les cinq années qui suivirent cette hausse spectaculaire, acceptée par le patronat pour arrêter la grève générale - selon la même logique qui avait prévalu à l'occasion de la grève générale du printemps 1936. La seconde "leçon" est politique. La plus grande grève générale de l'histoire européenne ne provoqua pas l'effondrement du régime et moins encore la révolution attendue par nombre des contestataires. Au contraire puisque l'UDR (Union pour la défense de la République, le parti gaulliste) remporta une écrasante victoire électorale les 23 et 30 juin 1968. Ce qui rappelle qu'un mouvement social, si puissant soit-il, ne peut déboucher sur une révolution qu'à la condition d'être associé à un mouvement politique qui lui donne les moyens d'atteindre concrètement les buts recherchés. Une « leçon » qui, en ce mois de décembre 2018 où je reprends le texte de ma conférence de juin dernier, marqué en France par le mouvement dit des «Gilets jaunes », semble plus que jamais valable...

\section{NOTES}

1. Par exemple, le numéro spécial de Beaux-Arts Magazine, paru en avril 2018 et titré « Mai 68. La révolution des images de $\mathrm{A}$ à $\mathrm{Z}$ ».

2. Dernière en date, aux Archives nationales (Hôtel de Soubise, à Paris) d'avril à septembre 2018 : «68. Les archives du pouvoir ». Le catalogue : ARTIÈRES, Philippe et GIRY, Emmanuelle (2018). 68. Les archives du pouvoir. Chroniques inédites d'un État face à la crise, Paris : L'Iconoclaste.

3. L'Institut national de l'audiovisuel a réédité en 2008 , avec l'aide du CNC et des éditions Montparnasse, plusieurs films tournés par des cinéastes solidaires du mouvement social, notamment ceux de Chris Marker dont certains produits par Slon-Iskra. 
4. Un exemple parmi beaucoup d'autres : Milou en mai de Louis Malle (1990). Le film donne une vision décalée bien que clairement socio-politique des événements, vécus dans le département rural du Gers par une famille bourgeoise qui se retrouve et se déchire autour d'un héritage.

5. Sur l'établissement, voir l'extraordinaire témoignage de Robert Linhart dans L'établi, paru aux éditions de Minuit en 1978. Et le témoignage de sa fille, Virginie Linhart Le Jour où mon père s'est $t u$, aux éditions du Seuil en 2008.

6. Le plus important: Les Années 68. Le temps de la contestation, organisé par Geneviève DreyfusArmand, Robert Frank, Marie-Françoise Lévy et Michelle Zancarini-Fournel, publié chez Complexe (Bruxelles) en 2000. En 1988, un premier colloque d'historiens et de politistes, 1968. Exploration du mai français, avait ouvert la voie, organisé par René Mouriaux, Annick Percheron, Antoine Prost et Danielle Tartakowsky. Il fut publié chez L'Harmattan en 1992.

7. Le principal par son ampleur de vue, dirigé par Philippe ARTIÈRES et Michelle ZANCARINIFOURNEL et titré 68. Une Histoire collective, paru aux éditions La Découverte en 2008.

8. Le plus original est sans doute l'ouvrage de témoignages (plusieurs centaines) coordonné par Christelle Dormoy-Rajramanan, Boris Gobille et Érik Neveu: Mai 68 par ceux qui l'ont vécu, publié par les éditions de L'Atelier et Médiapart.

9. Voir le beau livre de Christian Bougeard paru aux Presses universitaires de Rennes en 2018 et titré Les Années 68 en Bretagne. Les mutations d'une société (1962-1981).

10. Sur ce thème de l'insubordination ouvrière, le livre de référence est celui de Xavier Vigna, L'Insubordination ouvrière dans les années 1968. Essai d'histoire politique des usines, paru aux Presses universitaires de Rennes en 2007.

11. Christian Bougeard, Vincent Porhel, Gilles Richard et Jacqueline Sainclivier (dir.) L'Ouest dans les années 68, Presses universitaires de Rennes, 2009.

12. Parmi les nombreux ouvrages sur ce sujet, on peut lire celui de Florence Rochefort, Bibia Pavard et Michelle Zancarini-Fournel intitulé Les Lois Veil. Contraception 1974. IVG 1975, paru chez Armand Colin en 2012.

13. Sur cette question, voir les actes du colloque organisé par Gilles Richard et Jacqueline Sainclivier, Les Partis à l'épreuve de 68. L'émergence de bouveaux clivages, 1971-1974, publié aux Presses universitaires de rennes en 2012.

14. Des éléments dans le livre de Xavier Vigna déjà cité et dans celui de Gilles Richard intitulé Mai 68... et après? Une nouvelle donne politique, publié par le CRDP d'Aquitaine dans la colelction « Histoire de notre temps », en 2008.

15. Vue d'ensemble sur l'évolution politique dans Gilles Richard Histoire des droites en France de 1815 à nos jours, paru chez Perrin en 2017.

\section{RÉSUMÉS}

Le « moment 68 » français s'inscrivit dans un contexte mondial ouvert au début des années 1960 et marqué par des mouvements socio-culturels multiformes, toujours à forte connotation politique, dans lesquels la jeunesse occupa une place de choix. Mais il représenta en même temps un événement spécifique, sans commune mesure avec ce que les autres pays occidentaux connurent, Italie exceptée. En effet, les mois de mai et juin 1968 virent se dérouler en France une immense grève générale conduite (7 millions de grévistes) avec occupation des entreprises. Ce mouvement social atypique - largement oublié aujourd'hui - eut des conséquences 
contradictoires. Il ouvrit une période de luttes sociales tous azimuts (grèves de femmes et d'immigrés, deuxième féminisme, essor de l'écologie, antimilitarisme) pendant que communistes et socialistes concluaient une alliance politique comme en 1936. Mais les classes dirigeantes, conscientes des menaces qui pesaient sur le capitalisme et l'organisation de la société, s'activèrent à l'inverse pour trouver des contre-feux nécessaires pour sauvegarder l'ordre social, au prix d'un certain nombre de concessions que la victoire de v. Giscard d'Estaing aux présidentielles de 1974 permirent de mettre en œuvre.

The French «moment 68 ", is placed within a world-wide context which began in the early 1960s and was marked by multiple, strongly political, socio-cultural movements in which youth assumed a prominent position. Yet, at the same time, it also represented a specific event incommensurate with what other western countries experienced, with the exception of Italy. Indeed, May and June of 1968 were stage to a massive general strike (7 million strikers) along with the occupation of companies. This largely forgotten, atypical social movement had contradictory consequences. It led to an all-encompassing period of social struggle (women's and migrants' strikes, a second feminist wave, the development of environmentalism and antimilitarism), while communists and socialists struck up a political alliance as had occurred in 1936. However, the ruling classes, aware of the emerging threat to capitalism and to the organisation of civil society, strove to find countermeasures that safeguarded social order at the expense of a number of concessions implemented with v. Giscard d'Éstaing's 1974 victorious presidential elections.

\section{INDEX}

Mots-clés : clivage droites-gauches, contre-culture, grève générale, jeunesse, mouvements sociaux

Keywords : right-left division, counterculture, general strike, youth, social movements

\section{AUTEUR}

\section{GILLES RICHARD}

Université Rennes 2

gilles.richard[at]univ-rennes2.fr 